\title{
PASSENGER FLOW ANALYSIS IN RAILWAY TRANSIT STATION BY POTENTIAL PATHLINE METHOD
}

\author{
SEONGWON NAM \& JAEHO KWAK \\ Korea Railroad Research Institute, Korea
}

\begin{abstract}
The pedestrian flow model is a design tool that can be applied to railway transit stations, multi complex buildings, shopping malls, and stadiums. In this study, a new numerical analysis method was developed to simulate pedestrian flow. It is a computational analysis method using the concept of potential pathline. The domain to be analysed is divided into small calculation cells, and a potential value is gradually given starting from the exit cell. Based on the potential values, we can obtain the direction vector for each cell and draw the pathline following it. These pathlines are the basic routes for individual pedestrians to move between the origin and the destination. And then, pedestrian might changes the route according to the cell conditions of each location and moves to the destination. In this analytical method, visual angle, straightness probability, speed, and right-hand traffic can be used as parameters. It can also be applied to a three-dimensional interlayer movement model. Using the developed analysis program, we simulated about the passenger flow at the passageway of railway transit station and also compared Dirk Helbing's result (Nature 2000) and found that the results of both methods are in good agreement.
\end{abstract}

Keywords: pedestrian flow, computational analysis, railway station, potential pathline.

\section{INTRODUCTION}

Railway transit stations, multi-complex facilities, large shopping malls, and stadiums are typical urban structures where crowd flows occur. There are a number of factors that affect how people choose to travel route from the origin to the destination. If the path is known in advance, people will use the shortest path, but if it is a strange one, visibility for structure and various guide signs will work. In the case of railway transit stations, transit passengers facing in opposite directions face each other, so a typical bidirectional passenger flow occurs. Many methods have been proposed to study the crowd flow dynamics. Nam proposed a passenger flow analysis algorithm based on the Discrete Element Method [1], [2], Helbing used the social force model to study the movement of people in the panic state [3]-[5]. There are also examples using Cellular automata model [6]-[8], lattice gas model [9]-[12], Agent-base model [13], and Forces model [14]. Each of these analysis methods has advantages and disadvantages, and is appropriately applied according to the calculation model.

Passenger flow analysis method developed in this study can apply not only two-way passenger flow but also interlayer movement model. Individual trajectories were traced by the Lagrangian method. When a pedestrian selects a path, a pathline that follows a potential value for each computational grid is a basic path between ODs, and the alternative path is searched for and moved according to the state of the cell at each movement time. When looking for alternative routes, visibility, the probability of going straight, the percentage of fast and slow people, the speed of walking, and the right traffic are the parameters that determine the route. The results of the analysis are compared with Helbing's results and applied to the transit passage model.

\section{POTENTIAL PATHLINE METHOD}

The potential pathline method (PPM), which is the basic concept of this study, can be explained in Fig. 1. When small particles of sand are contained in the funnel, the particles 
tend to flow down through the outlet with the lowest potential energy. The potential energy increases in the order of 1,2,3, and 4, which can be defined as the potential in fluid mechanics. That is, they have the same potential value at the same distance from the outlet. Particles tend to move from large potential site to small ones. These tangential lines are pathlines, which are perpendicular to the equipotential lines. Assuming that the exit has the smallest potential value in the pedestrian flow domain, we can create a potential map for the entire analysis domain.

To illustrate the potential pathline method developed in this study, we set up the model as shown in Fig. 2(a). It is an analytical model in which people come in from the two entrances of a room with a wall in the middle and go out through one outlet. The definition of the coordinates of the cells used in the calculation is shown in Fig. 2(b). The calculation region is divided into cells of length DL, and I and $\mathrm{J}$ addresses are assigned to the cells in the $\mathrm{x}$ and y directions, respectively. Each cell can contain only one person, and the cell length DL can be set differently depending on the characteristics of the problem to be analyzed.

According to the concept of the potential pathline, a potential value 1 is set to the outlet cell, and a potential value is incremented by 1 for the adjacent cell. If the potential values are sequentially given to other cells as well, a potential map for the entire calculation region can be constructed. That is, if the potential value of cell $(\mathrm{I}, \mathrm{J})$ is $\mathrm{M}$, the potential value of the adjacent cell has $M+1$. In the potential map of Fig. 3(a), a pedestrian located in the
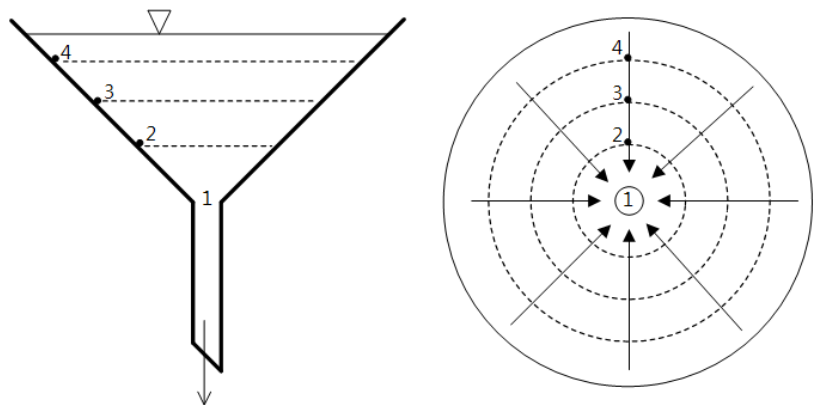

Figure 1: Concept of potential pathline method.

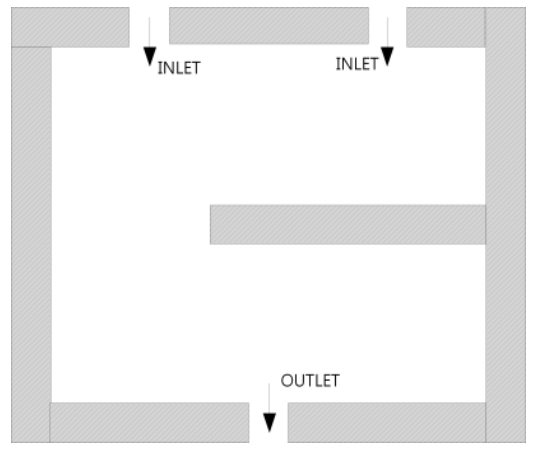

(a)

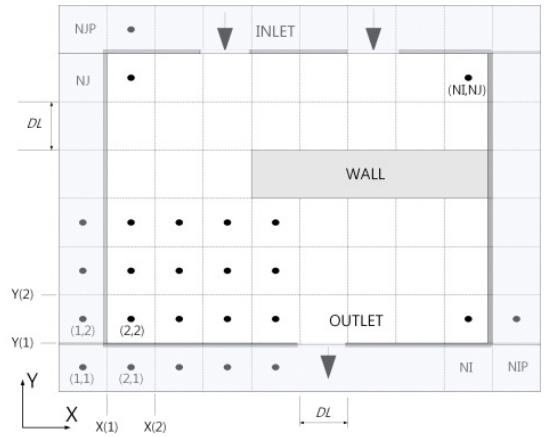

(b)

Figure 2: Analysis model and numerical cell. (a) Analysis model; (b) Numerical cell. 
(6) cell with a potential value of 6 tries to move from the adjacent cell to the cell with the potential value 5 . In this case, since there are three cells having a potential value of 5 , the potential values of the next cell are compared with each other to find the minimum value. That is, the cells in the lower right direction have values of 5, 4, and 3 . The cells in the right direction have values of 5, 4, and 4. The cells in the upper right direction have values of 5, 6, and 7, respectively. That is, in (6) cell, the direction vector is in the lower right direction. Fig. 3(b) shows a pathline that follows the direction vector for each cell and the direction vector in each cell. If the pedestrian is not disturbed, it can be predicted that pedestrians will move to the exit along each pathline.

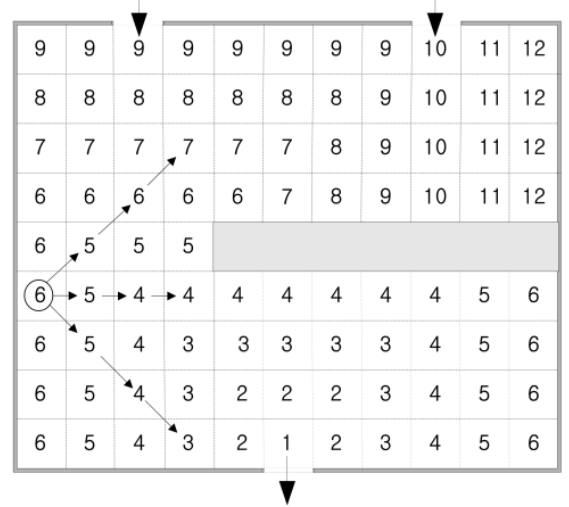

(a)

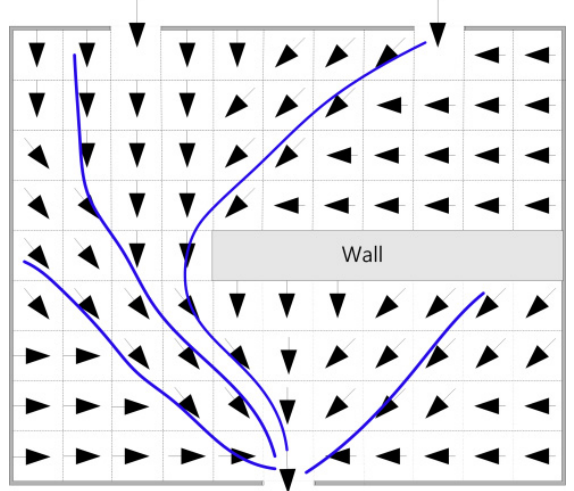

(b)

Figure 3: (a) Potential map; and (b) Pathline.

Figure 4: Shows the change of pedestrian position at each time along the pathline. After the unit time, the new position to move is shown in eqn (1).

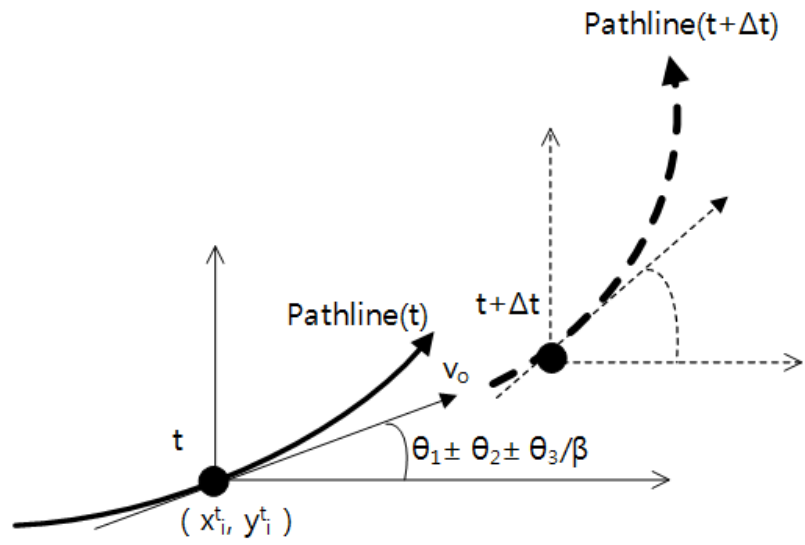

Figure 5: Change of pedestrian position at each time. 


$$
\overline{\boldsymbol{S}}_{i j}^{t+d t}=\left\{x_{i j}^{t}+\alpha V_{0} \cos \left(\theta_{1} \pm \theta_{2} \pm \frac{\theta_{3}}{\beta}\right) \Delta t\right\} \overline{\boldsymbol{I}}+\left\{y_{i j}^{t}+\alpha V_{0} \sin \left(\theta_{1} \pm \theta_{2} \pm \frac{\theta_{3}}{\beta}\right) \Delta t\right\} \overline{\boldsymbol{J}},
$$

where, $x_{i j}^{t}$ and $y_{i j}^{t}$ are positions in the $\mathrm{x}$ and $\mathrm{y}$ directions at time $\mathrm{t}, V_{0}$ is the standard walking speed, $\theta_{1}$ is the tangential angle of the pathline at $\mathrm{ij}, \theta_{2}$ is the angle deviating from the straight line according to the straightness probability, $\theta_{3}$ is the viewing angle, $\alpha$ is the pedestrian group number (specifying a slow pedestrian or a fast pedestrian), $\beta$ is the right-hand pass coefficient.

If there is an obstacle such as pedestrian or wall in a cell in the $\theta_{1}$ direction along the pathline at $x_{i j}^{t}$ and $y_{i j}^{t}$, then the pedestrian considers $\theta_{2}$ and $\theta_{3}$ sequentially to find vacant cells in the other direction. If the pedestrian has a high probability of going straight, $\theta_{2}$ is zero. In addition, if only the right traffic is possible, $\mathrm{x}$ is 2 since the selectable cells are limited to the right. If the pathline of the cell is the vertical direction in Fig. 5, the direction of movement is $(\mathrm{I}, \mathrm{J}+1)$ or $(\mathrm{I}, \mathrm{J}+2)$. If there is an obstacle in the direction of movement or another pedestrian, the pedestrian searches for an empty cell. Slower pedestrians can move to 8 cells in a small circle, and fast pedestrians can move to 16 cells across a large circle radius. A fast pedestrian in cell $(\mathrm{I}, \mathrm{J})$ tries to move to cell $(\mathrm{I}, \mathrm{J}+2)$ in unit time. If there is an obstacle in the cell, the pedestrian searches for an empty cell in the vicinity. If the viewing angle is $90^{\circ}$, the alternate cell is searched for four cells within the range of angle COC, ie, (I-1, J+2), (I+1, $\mathrm{J}+2),(\mathrm{I}-2, \mathrm{~J}+2)$, and $(\mathrm{I}+2, \mathrm{~J}+2)$. If an alternative cell is not found, the alternative cell is searched for two cells in a small circle, $(\mathrm{I}-1, \mathrm{~J}+1)$, and $(\mathrm{I}+1, \mathrm{~J}+1)$. If an alternative cell is not found among these cells, it is assumed to stay at the original position. If the viewing angle is $180^{\circ}$, the alternate cell is searched for eight cells within the range of angle AOA, ie, (I-2, J), $(\mathrm{I}-2, \mathrm{~J}+1),(\mathrm{I}-2, \mathrm{~J}+2),(\mathrm{I}-1, \mathrm{~J}+2),(\mathrm{I}+1, \mathrm{~J}+2),(\mathrm{I}+2, \mathrm{~J}+2),(\mathrm{I}+2, \mathrm{~J}+1)$, and $(\mathrm{I}+2, \mathrm{~J})$. If an alternative cell is not found, the alternative cell is searched for four cells in a small circle, (I$1, J),(I-1, J+1),(I+1, J+1)$, and $(I+1, J)$. That is, as the viewing angle increases, the number of alternative cells that can be found increases. If the pedestrian meets the opposite pedestrian, it is assumed that he is walking on the right side of each other. Since the viewing angle can be regarded as limited only to the right direction from the baseline, the alternative cell

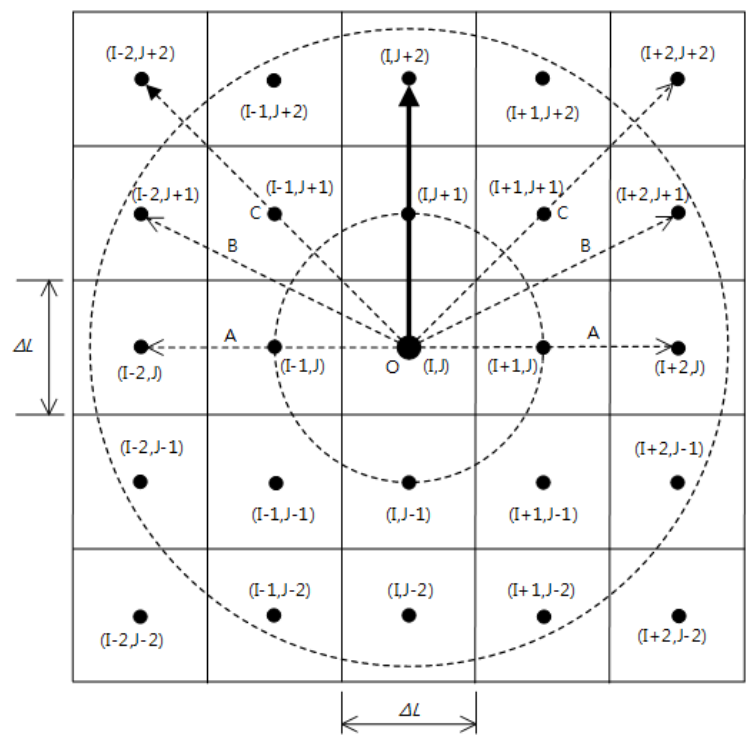

Figure 6: Alternative Cell Finding. 
searches only in the cells within right side. In general, pedestrians do not walk straightly in a desired direction, but slightly zigzag behaviors, so we introduced the effect of straight-line probability that deviates slightly from the original target direction.

\section{RESULTS AND DISCUSSION}

In order to compare the validity and performance of this method, the results of Helbing were taken as an example. 200 people in a room of $15 \mathrm{~m} \times 15 \mathrm{~m}$ go out at a speed of $1 \mathrm{~m} / \mathrm{s}$ through $1 \mathrm{~m}$ wide exit on the right. Fig. 6 shows a snapshot of the results. The crowd flow characteristics in this model are arching and clogging around the exit. Both analytical results are qualitatively similar. The result of the Helbing's paper shows that the arching is made at about the same radius around the exit. Since Helbing uses the social force model, it seems to be overly symmetrical.

90 people in a room of $15 \mathrm{~m} \times 15 \mathrm{~m}$ go out at a speed of $5 \mathrm{~m} / \mathrm{s}$ through $1.5 \mathrm{~m}$ wide exit on both sides. The characteristics of the analysis results for this model are as follows: the crowd is not distributed evenly on both left and right sides due to the herding phenomenon. The analytical results are similar to each other.

The analytical model is a linkage that is commonly seen in transit stations, and it has a shape bent to the left and right. Fig. 8 is the snapshot of result for the connection channel model of railway transit station. Fig. 9 shows the movement trajectories of passengers. The group of red circle sign enters from the left side and goes to the right side, and the group of blue triangle sign enters from the right side to the left side at a rate of 1 person $/ \mathrm{ms}$ for 10 seconds. As a result of walking on the right, Fig. 8(b), the group of red circle sign is distributed on the right side and the group of blue triangle sign is distributed on the left side at the middle intersection area. In Fig. 8(a), we can see that the two groups are randomly

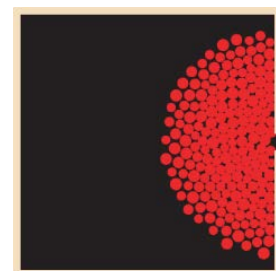

(a)

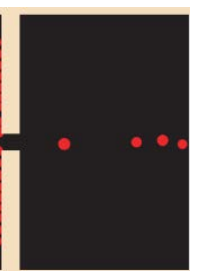

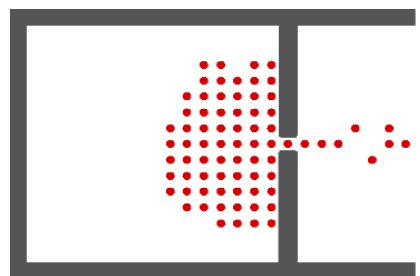

(b)

Figure 7: Comparison with Helbing's result and present (1). (a) Helbing's result [3]; (b) Present.

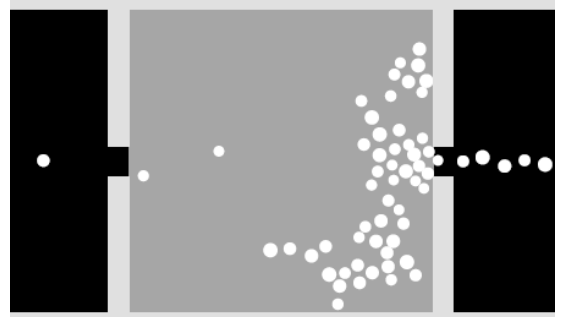

(a)

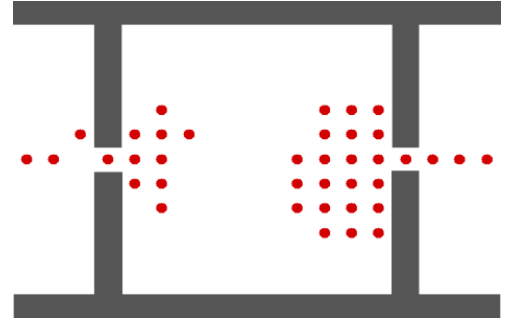

(b)

Figure 8: Comparison with Helbing's result and present (2). (a) Helbing's result [3]; (b) Present. 


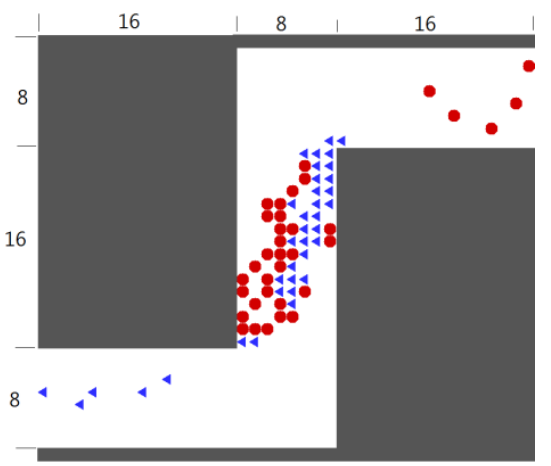

(a)

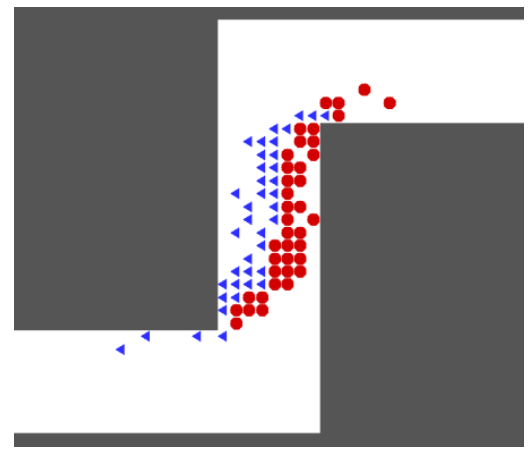

(b)

Figure 9: Comparison of snapshot. (a) W/o right-hand traffic; (b) Right-hand traffic.

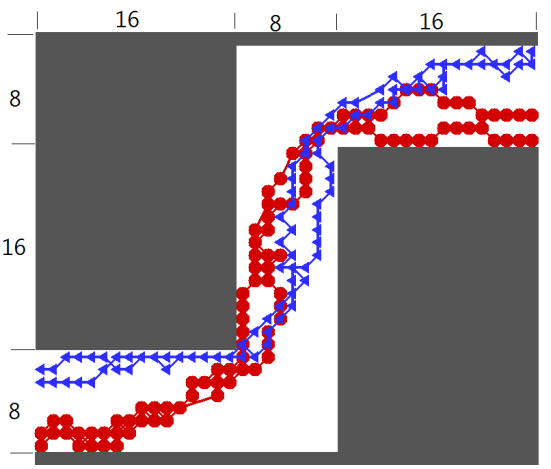

(a)

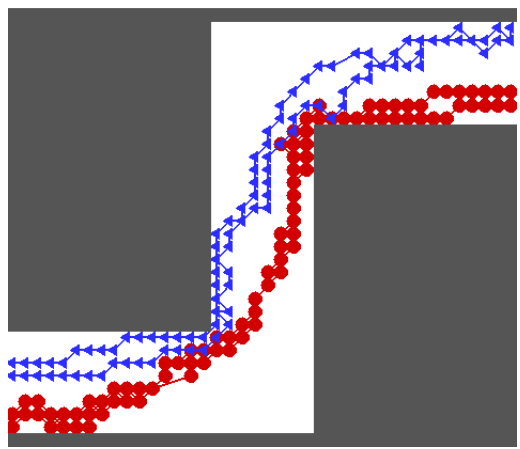

(b)

Figure 10: Comparison of trajectory. (a) W/o right-hand traffic; (b) Right-hand traffic.

mixed. The results of right-hand traffic can also be seen in Fig 9. As shown in Fig. 9(b), there is no crossing of the trajectory at the center intersection because the two groups hold the right side at the inflow, middle and exit parts. In Fig. 9(a), however, the trajectory between the two groups overlaps at the middle part.

\section{CONCLUSIONS}

The pedestrian flow analysis is a design tool that can be applied to railway transit stations, multi complex buildings, shopping malls, and stadiums. In this study, a new numerical analysis method was developed to simulate pedestrian flow. It is a computational analysis method using the concept of potential-pathline. The domain to be analysed is divided into small calculation cells, and a potential value is gradually given starting from the exit cell. Based on the potential values, we can obtain the direction vector for each cell and draw the pathline following it. These pathlines are the basic routes for individual pedestrians to move between the origin and the destination. And then, pedestrian might changes the route according to the cell conditions of each location and moves to the destination. In this analytical method, visual angle, straightness probability, speed, and right-hand traffic can be used as parameters. Using the developed analysis program, we simulated about the passenger 
flow at the passageway of railway transit station and also compared Dirk Helbing's result and found that the results of both methods are in good agreement. As a result of the analysis, it was possible to review the influence of the visual angle and the right-hand traffic in the passage model of railway transit station.

\section{ACKNOWLEDGEMENT}

This research was supported by a grant (DR18001 and PK1802B2B) funded by Ministry of Land, Infrastructure and Transport of Korean government.

\section{REFERENCES}

[1] Nam, S., Development of algorithm for passenger flow analysis based on DEM. Journal of the Korean Society for Railway, 8(4), pp. 337-341, 2005.

[2] Nam, S., Analysis of pedestrian flow characteristics in subway station. Journal of the Korean Society for Railway, 9(3), pp. 271-276, 2006.

[3] Helbing, D., Farkas, I. \& Vicsek, T., Simulating dynamical features of escape panic. Nature, 407(28), pp. 487-490, 2000.

[4] Helbing, D., Molnar, P., Social force model for pedestrian dynamics. Physical Review E, 51, pp. 4282-4286, 1995.

[5] Seyfried, A., Steffen, B. \& Lippert, T., Basics of modelling the pedestrian flow. Physica A, 368, pp. 232-238, 2006.

[6] Nagal, K. \& Schreckenber, M., A cellular automation model for freeway traffic. Journal of Physics, 2(12), pp. 2221-2229, 1992.

[7] Burstedde, C., Klauck, K. \& Schadschneider, A., Simulation of pedestrian dynamics using a two-dimensional cellular automaton. Physica A, 295, pp. 507-525, 2001.

[8] Blue, V. \& Adler, J., Cellular automata microsimulation for modelling bi-directional pedestrian walkways. Transportation Research Part B, 35, pp. 293-312, 2001.

[9] Helbing, D., Isobe, M., Nagatani, T. \& Takimoto, K., Lattice gas simulation of experimentally studied evacuation dynamics. Physical Review E, 67, 067101, pp. 1-4, 2003.

[10] Guo, R. \& Huang, H., A mobile lattice gas model for simulating pedestrian evacuation. Physica A, 387, pp. 580-586, 2008.

[11] Antonini, G., Bierlaire, M. \& Weber, M., Discrete choice models of pedestrian walking behavior. Transportation Research Part B, 40, pp. 667-687, 2006.

[12] Singh, H., Arter, R., Dodd, L., Langston, P., Lester, E. \& Drury, J., Modelling subgroup behavior in crowd dynamics DEM simulation. Applied Mathematical Modelling, 33, pp. 4408-4423, 2009.

[13] Heliovaara, S., Korhonen, T., Hostikka, S. \& Ehtamo, H., Counterflow model for agebt-based simulation of crowd dynamics. Building and Environment, 48, pp. 89$100,2012$.

[14] Lohner, R., On the modelling of pedestrian motion. Applied Mathematical Modelling, 34, pp. 366-382, 2010. 